\title{
On the new double integral transform for solving singular system of hyperbolic equations
}

\author{
A. A. Alderremy ${ }^{a, *}$, Tarig. M. Elzakib,c \\ ${ }^{a}$ Mathematics Department, Faculty of Sciences, King Kalied University, Abha, Saudi Arabia. \\ ${ }^{b}$ Mathematics Department, Faculty of Sciences and Arts-Alkamil, University of Jeddah, Jedda, Saudi Arabia. \\ ${ }^{c}$ Mathematics Department, Faculty of Sciences, Sudan University of Sciences and Technology, Sudan.
}

Communicated by R. Saadati

\begin{abstract}
In this manuscript, we will introduce a new double transform called double Elzaki transform (modification of Smudu transform), where we will study this transform and their theorems on convergence. Also, we will discuss the double new transform and it is convergent. After that, we study the combination of this double transforms and the new method in order to solve the singular system of hyperbolic equations of anomalies in through the examples in this paper. We found that this method is very effective in solving these equations compared to other methods as they need only one step to get the exact solution, while the other methods need more steps.
\end{abstract}

Keywords: Double new integral, transform, convergence, nonlinear singular system of hyperbolic equations.

2010 MSC: 35A22, 35L81.

(C)2018 All rights reserved.

\section{Introduction}

Nonlinear equations are of great importance to our contemporary world. Nonlinear phenomena have important applications in applied mathematics, physics, and issues related to engineering. Despite the importance of obtaining the exact solution of nonlinear partial differential equations in physics and applied mathematics there is still the daunting problem of finding new methods to discover new exact or approximate solutions. In the recent years, many authors have devoted their attention to study solutions of nonlinear partial differential equations using various methods. Among these attempts are the Adomian decomposition method, homotopy perturbation method, variational iteration method [1, 8-10], Laplace variational iteration method [12, 13, 22] differential transform method, Elzaki transform [2, 3, 6], Laplace, double Laplace transforms $[2,4]$ and projected differential transform method. Many analytical and numerical methods have been proposed to obtain solutions for nonlinear PDEs with fractional derivatives,

\footnotetext{
*Corresponding author

Email addresses: aaldramy@kku.edu.sa (A. A. Alderremy), tfarah@ uj.edu.sa and tarig.alzaki@gmail.com (Tarig. M. Elzaki)
}

doi: $10.22436 /$ jnsa.011.10.08

Received: 2018-04-17 Revised: 2018-06-05 Accepted: 2018-06-28 
such as local fractional variational iteration method [11], local fractional Fourier method, Yang-Fourier transform and Yang-Laplace transform and other methods [14-18]. Two Laplace variational iteration methods are currently suggested by $\mathrm{Wu}$ in [19-21, 23]. In this paper, we will introduce the new method depends on double new integral transform (double Elzaki transform) [7], and it will be employed in a straightforward manner. Also, we study in this paper the combination of this new transform and the new method to solve the singular system of hyperbolic equations. This approach can be taken functions with discontinuities as well as impulse functions effectively.

Elzaki transform, henceforth designated by the operator $E[$.$] , is defined by the integral equation,$

$$
E[\Omega(t)]=T(\beta)=\beta^{2} \int_{0}^{\infty} \Omega(\beta t) e^{-t} d t .
$$

By analogy with the double Laplace transform, we shall denote the double Elzaki transform.

\section{New double integral transform}

In this section and analogy with the double Laplace transform, we will denote the new double transform. Also, in this paper, we will see the importance of this new double transform and its effectiveness in solving some differential equations.

Definition 2.1. Let $\Omega(x, t), t, x \in R^{+}$, be a function which can be expressed as a convergent infinite series, then its new double integral transform given by

$$
E_{2}[\Omega(x, t), \alpha, \beta]=T(\alpha, \beta)=\alpha \beta \int_{0}^{\infty} \int_{0}^{\infty} \Omega(x, t) e^{-\left(\frac{x}{\alpha}+\frac{t}{\beta}\right)} d x d t, \quad x, t>0,
$$

where $\alpha, \beta$ are complex values.

To find the solution of the singular system of hyperbolic equations by the combination of new double transform and the new method, first we must find the new double transform of partial derivatives as follows:

$$
\begin{aligned}
& E_{2}\left[\frac{\partial \Omega}{\partial x}\right]=\frac{1}{\alpha} \mathrm{T}(\alpha, \beta)-\alpha \mathrm{T}(0, \beta), \quad \mathrm{E}_{2}\left[\frac{\partial^{2} \Omega}{\partial x^{2}}\right]=\frac{1}{\alpha^{2}} \mathrm{~T}(\alpha, \beta)-\mathrm{T}(0, \beta)-\alpha \frac{\partial \mathrm{T}(0, \beta)}{\partial x}, \\
& E_{2}\left[\frac{\partial \Omega}{\partial t}\right]=\frac{1}{\beta} \mathrm{T}(\alpha, \beta)-\beta \mathrm{T}(\alpha, 0), \quad \mathrm{E}_{2}\left[\frac{\partial^{2} \Omega}{\partial t^{2}}\right]=\frac{1}{\beta^{2}} \mathrm{~T}(\alpha, \beta)-\mathrm{T}(\alpha, 0)-\beta \frac{\partial \mathrm{T}(\alpha, 0)}{\partial t}, \\
& E_{2}\left[\frac{\partial^{2} \Omega}{\partial x \partial t}\right]=\frac{1}{\alpha \beta} \mathrm{T}(\alpha, \beta)-\frac{\beta}{\alpha} \mathrm{T}(\alpha, 0)-\frac{\alpha}{\beta} \mathrm{T}(0, \beta)+\alpha \beta \mathrm{T}(0,0) .
\end{aligned}
$$

Proof.

$$
E_{2}\left[\frac{\partial \Omega}{\partial x}\right]=\alpha \beta \int_{0}^{\infty} \int_{0}^{\infty} e^{-\left(\frac{x}{\alpha}+\frac{t}{\beta}\right)} \frac{\partial}{\partial x} \Omega(x, t) d x d t=\beta \int_{0}^{\infty} e^{-\frac{t}{\beta}}\left\{\alpha \int_{0}^{\infty} e^{-\frac{x}{\alpha}} \frac{\partial}{\partial x} \Omega(x, t) d x\right\} d t .
$$

The inner integral gives $\frac{1}{\alpha} \mathrm{T}(\alpha, \mathrm{t})-\alpha \Omega(0, \mathrm{t})$, and then:

$$
E_{2}\left[\frac{\partial \Omega}{\partial x}\right]=\frac{\alpha}{\beta} \int_{0}^{\infty} e^{-\frac{t}{\beta}} T(\alpha, t) d t-\alpha \beta \int_{0}^{\infty} e^{-\frac{t}{\beta}} \Omega(0, t) d t=\frac{1}{\alpha} T(\alpha, \beta)-\alpha \mathrm{T}(0, \beta) .
$$

Also,

$$
E_{2}\left[\frac{\partial \Omega}{\partial t}\right]=\frac{1}{\beta} T(\alpha, \beta)-\beta T(\alpha, 0) .
$$

We can prove the formulas mentioned in (2.2) easily by using the same method. 


\section{Theorems of convergence of double new integral transform}

Here we need to discuss some theorems of convergence of double new integral transform.

Theorem 3.1. Suppose that $\beta \int_{0}^{\infty} e^{-\frac{t}{\beta}} \Omega(x, t) d t$, converges at $\beta=\beta_{0}$, then the integral converges at $\beta<\beta_{0}$. Proof. Let

$$
p(x, t)=\beta_{0} \int_{0}^{t} e^{-\frac{u}{\beta_{0}}} \Omega(x, u) d u, \quad 0<t<\infty,
$$

then we have

(i) $p(x, 0)=0$,

(ii) $\lim _{\mathrm{t} \rightarrow \infty} \mathrm{p}(\mathrm{x}, \mathrm{t})$ exist,

(iii) $p_{t}(x, t)=\beta_{0} e^{-\frac{t}{\beta_{0}}} \Omega(x, t)$.

Choosing $\varepsilon_{1}, R_{1}$, such that $0<\varepsilon_{1}<R_{1}$, then we have

$$
\beta \int_{\varepsilon_{1}}^{R_{1}} e^{-\frac{t}{\beta}} \Omega(x, t) d t=\beta \int_{\varepsilon_{1}}^{R_{1}} \frac{1}{\beta_{0}} e^{-\frac{t}{\beta}} P_{t}(x, t) e^{\frac{t}{\beta_{0}}} d t=\frac{\beta}{\beta_{0}} \int_{\varepsilon_{1}}^{R_{1}} e^{-\left(\frac{\beta_{0}-\beta}{\beta \beta_{0}}\right)} P_{t}(x, t) d t .
$$

Integrating the last integral by parts to gives

$$
\begin{aligned}
\frac{\beta}{\beta_{0}} \int_{\varepsilon_{1}}^{R_{1}} e^{-\left(\frac{\beta_{0}-\beta}{\beta \beta_{0}}\right) t} P_{t}(x, t) d t= & \frac{\beta}{\beta_{0}}\left\{e^{-\left(\frac{\beta_{0}-\beta}{\beta \beta_{0}}\right) R_{1}} p\left(x, R_{1}\right)-e^{-\left(\frac{\beta_{0}-\beta}{\beta \beta_{0}}\right) \varepsilon_{1}} p\left(x, \varepsilon_{1}\right)\right. \\
& \left.+\left(\frac{\beta_{0}-\beta}{\beta \beta_{0}}\right) \int_{\varepsilon_{1}}^{R_{1}} e^{-\left(\frac{\beta_{0}-\beta}{\beta \beta_{0}}\right)} p(x, t) d t\right\} .
\end{aligned}
$$

Now take, $\varepsilon_{1} \rightarrow 0, R_{1} \rightarrow \infty$, and if $\beta<\beta_{0}$, then we have

$$
\beta \int_{0}^{\infty} \Omega(x, t) d t=\frac{\beta_{0}-\beta}{\beta \beta_{0}} \int_{0}^{\infty} e^{-\left(\frac{\beta_{0}-\beta}{\beta \beta_{0}}\right)} p(x, t) d t, \quad \beta<\beta_{0} .
$$

Theorem 3.1 is proved if the last integral is converges.

By using the limits test for convergence we get

$$
\lim _{t \rightarrow \infty} t^{2} e^{-\left(\frac{\beta_{0}-\beta}{\beta \beta_{0}}\right) t} p(x, t)=0,
$$

finite. Therefore

$$
\beta \int_{0}^{\infty} e^{-\frac{t}{\beta}} \Omega(x, t) d t
$$

is converges for $\beta<\beta_{0}$.

Theorem 3.2. Let the integral $\mathrm{Q}(x, \beta)=\beta \int_{0}^{\infty} e^{-\frac{\mathrm{t}}{\beta}} \Omega(x, \mathrm{t}) \mathrm{dt}$ converges for $\beta<\beta_{0}$ and the integral

$$
\alpha \int_{0}^{\infty} e^{-\frac{x}{\alpha}} \Omega(x, \beta) d x
$$

converges at $\alpha=\alpha_{0}$. Then the integral $\alpha \int_{0}^{\infty} e^{-\frac{x}{\alpha}} \mathrm{Q}(x, \beta) \mathrm{d} x$ converges for $\alpha<\alpha_{0}$.

Proof. The prove of this theorem is same as the method in Theorem 3.1

Theorem 3.3. Let the function $\Omega(x, t)$ is continuous in the $x y$-plane, if the integral converges for $\beta=\beta_{0}, \alpha=\alpha_{0}$. Then the integral

$$
\alpha \beta \int_{0}^{\infty} \int_{0}^{\infty} e^{-\frac{x}{\alpha}-\frac{t}{\beta}} \Omega(x, t) d x d t
$$

is converges for $\alpha<\alpha_{0}, \beta<\beta_{0}$. 
Proof.

$$
\alpha \beta \int_{0}^{\infty} \int_{0}^{\infty} e^{-\frac{x}{\alpha}-\frac{t}{\beta}} \Omega(x, t) d x d t=\alpha \int_{0}^{\infty} e^{-\frac{x}{\alpha}}\left\{\beta \int_{0}^{\infty} e^{-\frac{t}{\beta}} \Omega(x, t) d t\right\} d x=\alpha \int_{0}^{\infty} e^{-\frac{x}{\alpha}} Q(x, \beta) d x,
$$

where $Q(x, \beta)=\beta \int_{0}^{\infty} e^{-\frac{t}{\beta}} \Omega(x, t) d t$. By using Theorems 3.1 and 3.2 we see that

$$
\alpha \beta \int_{0}^{\infty} \int_{0}^{\infty} e^{-\frac{x}{\alpha}-\frac{t}{\beta}} \Omega(x, t) d x d t
$$

is converges for $\alpha<\alpha_{0}, \beta<\beta_{0}$.

\section{The new method with new double integral transform}

To explain this method we will display the singular system of hyperbolic equations,

$$
\begin{gathered}
\frac{\partial^{2} P}{\partial t^{2}}-\frac{1}{x} \frac{\partial}{\partial x}\left(x \frac{\partial P}{\partial x}\right)-Q=k(x, t), \\
\frac{\partial^{2} Q}{\partial t^{2}}-\frac{1}{x} \frac{\partial}{\partial x}\left(x \frac{\partial Q}{\partial x}\right)-P=h(x, t) .
\end{gathered}
$$

With the initial conditions

$$
\begin{array}{ll}
P(x, 0)=k_{1}(x), & P_{t}(x, 0)=k_{2}(x), \\
Q(x, 0)=h_{1}(x), & Q_{t}(x, 0)=h_{2}(x) .
\end{array}
$$

To find the solution of the system (4.1), (4.2), firstly we take double new integral transform of (4.1), and single new integral transform of (4.2), we obtain

$$
\begin{aligned}
& \frac{1}{\beta^{2}} E_{2}(P(x, t))-K_{1}(\alpha)-\beta K_{2}(\alpha)=E_{2}\left[k(x, t)+\frac{1}{x} \frac{\partial}{\partial x}\left(x \frac{\partial P}{\partial x}\right)+Q\right], \\
& \frac{1}{\beta^{2}} E_{2}(Q(x, t))-H_{1}(\alpha)-\beta H_{2}(\alpha)=E_{2}\left[h(x, t)+\frac{1}{x} \frac{\partial}{\partial x}\left(x \frac{\partial Q}{\partial x}\right)+P\right],
\end{aligned}
$$

where $K_{1}(\alpha), K_{2}(\alpha), H_{1}(\alpha), H_{2}(\alpha)$, are single new integral transform of $k_{1}(x), k_{2}(x), h_{1}(x), h_{2}(x)$, respectively.

We assume that the solution of a system (4.1) can be written in the series form

$$
\mathrm{P}(\mathrm{x}, \mathrm{t})=\sum_{\mathrm{n}=0}^{\infty} \mathrm{P}_{\mathrm{n}}(\mathrm{x}, \mathrm{t}), \quad \mathrm{Q}(\mathrm{x}, \mathrm{t})=\sum_{\mathrm{n}=0}^{\infty} \mathrm{Q}_{\mathrm{n}}(\mathrm{x}, \mathrm{t}) .
$$

Now, we take the inverse of double new integral transform of (4.3), and making use of (4.4) to get

$$
\begin{aligned}
& \sum_{n=0}^{\infty} P_{n}(x, t)=k_{1}(x)+t k_{2}(x)+E_{2}^{-1}\left\{\beta^{2} E_{2}\left[k(x, t)+\frac{1}{x} \frac{\partial}{\partial x}\left(x \frac{\partial P_{n}}{\partial x}\right)+Q_{n}\right]\right\}, \\
& \sum_{n=0}^{\infty} Q_{n}(x, t)=h_{1}(x)+t_{2}(x)+E_{2}^{-1}\left\{\beta^{2} E_{2}\left[h(x, t)+\frac{1}{x} \frac{\partial}{\partial x}\left(x \frac{\partial Q_{n}}{\partial x}\right)+P_{n}\right]\right\} .
\end{aligned}
$$

This method depends on how to choose the initial iterations, $P_{0}(x, t), Q_{0}(x, t)$, that leads to the exact solutions in a few steps. For example if we choose

$$
P_{0}(x, t)=k_{1}(x)+t k_{2}(x), \quad Q_{0}(x, t)=h_{1}(x)+t_{2}(x) .
$$


Then the solutions $P(x, t), Q(x, t)$ can be recursively determined by using the relations

$$
\begin{aligned}
P_{n+1}(x, t) & =k_{1}(x)+t k_{2}(x)+E_{2}^{-1}\left\{\beta^{2} E_{2}\left[k(x, t)+\frac{1}{x} \frac{\partial}{\partial x}\left(x \frac{\partial P_{n}}{\partial x}\right)+Q_{n}\right]\right\} \\
\operatorname{Po}(x, t) & =k_{1}(x)+t k_{2}(x), \\
Q_{n+1}(x, t) & =h_{1}(x)+t_{2}(x)+E_{2}^{-1}\left\{\beta^{2} E_{2}\left[h(x, t)+\frac{1}{x} \frac{\partial}{\partial x}\left(x \frac{\partial Q_{n}}{\partial x}\right)+P_{n}\right]\right\}, \\
Q_{0}(x, t) & =h_{1}(x)+t_{2}(x) .
\end{aligned}
$$

From these equations we can find

$$
P_{0}(x, t), P_{1}(x, t), P_{2}(x, t), \cdots, \quad Q_{0}(x, t), Q_{1}(x, t), Q_{2}(x, t), \cdots,
$$

and then we can obtain the solutions in a series form (4.4).

\section{Application}

To illustrate the efficiency and effectiveness of this method in solving the singular system of linear and nonlinear hyperbolic equations by taking only one step, we look at the following examples.

Example 5.1. Let us consider the singular system of linear hyperbolic equations

$$
\begin{aligned}
& -\frac{\partial^{2} P}{\partial t^{2}}+\frac{1}{x} \frac{\partial}{\partial x}\left(x \frac{\partial P}{\partial x}\right)+Q=x^{2} \sin t+4 \sin t+x^{2} \cos t \\
& -\frac{\partial^{2} Q}{\partial t^{2}}+\frac{1}{x} \frac{\partial}{\partial x}\left(x \frac{\partial Q}{\partial x}\right)+P=x^{2} \sin t+4 \cos t+x^{2} \cos t
\end{aligned}
$$

with the initial conditions

$$
\mathrm{P}(x, 0)=0, \mathrm{P}_{\mathrm{t}}(x, \mathrm{o})=x^{2}, \mathrm{Q}(\mathrm{x}, 0)=x^{2}, \mathrm{Q}_{\mathrm{t}}(\mathrm{x}, 0)=0 .
$$

Using the same steps in Section 3 to get

$$
\begin{aligned}
& \frac{1}{\beta^{2}} E_{2}(P(x, t))-K_{1}(\alpha)-\beta K_{2}(\alpha)=-E_{2}\left[x^{2} \sin t\right]+E_{2}\left[\frac{1}{x} \frac{\partial}{\partial x}\left(x \frac{\partial P}{\partial x}\right)+Q-4 \sin t-x^{2} \cos t\right], \\
& \frac{1}{\beta^{2}} E_{2}(Q(x, t))-H_{1}(\alpha)-\beta H_{2}(\alpha)=-E_{2}\left[x^{2} \cos t\right]+E_{2}\left[\frac{1}{x} \frac{\partial}{\partial x}\left(x \frac{\partial Q}{\partial x}\right)+P-x^{2} \sin t-4 \cos t\right],
\end{aligned}
$$

where, $\mathrm{K}_{1}(\alpha)=0, \mathrm{~K}_{2}(\alpha)=2 \alpha^{4}, \mathrm{H}_{1}(\alpha)=2 \alpha^{4}, \mathrm{H}_{2}(\alpha)=0$. Then, (5.3) becomes

$$
\begin{aligned}
& E_{2}(P(x, t))=2 \beta^{3} \alpha^{4}-\frac{2 \beta^{5} \alpha^{4}}{1+\beta^{2}}+\beta^{2} E_{2}\left[\frac{1}{x} \frac{\partial}{\partial x}\left(x \frac{\partial P}{\partial x}\right)+Q-4 \sin t-x^{2} \cos t\right], \\
& E_{2}(Q(x, t))=2 \beta^{2} \alpha^{4}-\frac{2 \beta^{4} \alpha^{4}}{1+\beta^{2}}+\beta^{2} E_{2}\left[\frac{1}{x} \frac{\partial}{\partial x}\left(x \frac{\partial Q}{\partial x}\right)+P-x^{2} \sin t-4 \cos t\right] .
\end{aligned}
$$

Applying the inverse double new transform to (5.4), to obtain

$$
\begin{aligned}
& P(x, t)=x^{2} \sin t+E_{2}^{-1}\left\{\beta^{2} E_{2}\left[\frac{1}{x} \frac{\partial}{\partial x}\left(x \frac{\partial P}{\partial x}\right)+Q-4 \sin t-x^{2} \cos t\right]\right\}, \\
& Q(x, t)=x^{2} \cos t+E_{2}^{-1}\left\{\beta^{2} E_{2}\left[\frac{1}{x} \frac{\partial}{\partial x}\left(x \frac{\partial Q}{\partial x}\right)+P-4 \cos t-x^{2} \sin t\right]\right\} .
\end{aligned}
$$


Then the recursive relations are

$$
\begin{aligned}
P_{n+1}(x, t) & =E_{2}^{-1}\left\{\beta^{2} E_{2}\left[\frac{1}{x} \frac{\partial}{\partial x}\left(x \frac{\partial P_{n}}{\partial x}\right)+Q_{n}-4 \sin t-x^{2} \cos t\right]\right\}, \\
P_{0}(x, t) & =x^{2} \sin t, \\
Q_{n+1}(x, t) & =E_{2}^{-1}\left\{\beta^{2} E_{2}\left[\frac{1}{x} \frac{\partial}{\partial x}\left(x \frac{\partial Q_{n}}{\partial x}\right)+P_{n}-4 \cos t-x^{2} \sin t\right]\right\}, \\
Q_{0}(x, t) & =x^{2} \cos t .
\end{aligned}
$$

The first few components are given by

$$
\begin{aligned}
& P_{0}(x, t)=x^{2} \sin t, \\
& P_{1}(x, t)=E^{-1}\left\{\beta^{2} E_{2}[0]\right\}=0, \\
& Q_{0}(x, t)=x^{2} \cos t, \\
& Q_{1}(x, t)=E^{-1}\left\{\beta^{2} E_{2}[0]\right\}=0,
\end{aligned}
$$

Then the exact solutions of a system (5.1) are

$$
\mathrm{P}(\mathrm{x}, \mathrm{t})=\sum_{\mathrm{n}=0}^{\infty} \mathrm{P}_{\mathrm{n}}(\mathrm{x}, \mathrm{t})=\mathrm{x}^{2} \sin \mathrm{t}, \quad \mathrm{Q}(\mathrm{x}, \mathrm{t})=\sum_{n=0}^{\infty} \mathrm{Q}_{\mathrm{n}}(\mathrm{x}, \mathrm{t})=\mathrm{x}^{2} \cos \mathrm{t} .
$$

Example 5.2. Here we look at the singular system of nonlinear hyperbolic equations,

$$
\begin{aligned}
& -\frac{\partial^{2} \mathrm{P}}{\partial \mathrm{t}^{2}}+\frac{1}{x} \frac{\partial}{\partial x}\left(x \frac{\partial P}{\partial x}\right)+\mathrm{Q} \frac{\partial \mathrm{P}}{\partial x}=2 x e^{\mathrm{t} P}+4 \mathrm{t}, \\
& -\frac{\partial^{2} \mathrm{Q}}{\partial \mathrm{t}^{2}}+\frac{1}{x} \frac{\partial}{\partial x}\left(x \frac{\partial \mathrm{Q}}{\partial x}\right)+\mathrm{P} \frac{\partial \mathrm{Q}}{\partial x}=2 x \mathrm{tQ}-x^{2} e^{\mathrm{t}}+4 e^{\mathrm{t}},
\end{aligned}
$$

with the initial conditions

$$
\mathrm{P}(x, 0)=0, \mathrm{P}_{\mathrm{t}}(x, 0)=x^{2}, \mathrm{Q}(x, 0)=x^{2}, \mathrm{Q}_{\mathrm{t}}(x, 0)=x^{2} .
$$

Here we use the same steps which we used as before in Example 5.1, to obtain

$$
\begin{aligned}
& E_{2}(P(x, t))=2 \beta^{3} \alpha^{4}+\beta^{2} E_{2}\left[\frac{1}{x} \frac{\partial}{\partial x}\left(x \frac{\partial P}{\partial x}\right)+Q \frac{\partial P}{\partial x}-2 x e^{t} P-4 t\right], \\
& E_{2}(Q(x, t))=2 \beta^{2} \alpha^{4}+2 \beta^{3} \alpha^{4}+\beta^{2} E_{2}\left[x^{2} e^{t}\right]+\beta^{2} E_{2}\left[\frac{1}{x} \frac{\partial}{\partial x}\left(x \frac{\partial Q}{\partial x}\right)+P \frac{\partial Q}{\partial x}-2 x t Q-4 e^{t}\right] .
\end{aligned}
$$

Taking the inverse double new transform of (5.9), to find

$$
\begin{aligned}
& P(x, t)=x^{2} t+E_{2}^{-1}\left\{\beta^{2} E_{2}\left[\frac{1}{x} \frac{\partial}{\partial x}\left(x \frac{\partial P}{\partial x}\right)+Q \frac{\partial P}{\partial x}-2 x e^{t} P-4 t\right]\right\}, \\
& Q(x, t)=x^{2} e^{t}+E_{2}^{-1}\left\{\beta^{2} E_{2}\left[\frac{1}{x} \frac{\partial}{\partial x}\left(x \frac{\partial Q}{\partial x}\right)+P \frac{\partial Q}{\partial x}-2 x t Q-4 e^{t}\right]\right\} .
\end{aligned}
$$

Therefore, we can write the recursive relations as

$$
\begin{aligned}
P_{n+1}(x, t) & =E_{2}^{-1}\left\{\beta^{2} E_{2}\left[\frac{1}{x} \frac{\partial}{\partial x}\left(x \frac{\partial P_{n}}{\partial x}\right)+Q_{n} \frac{\partial P_{n}}{\partial x}-2 x e^{t} P_{n}-4 t\right]\right\}, \\
P_{0}(x, t) & =x^{2} t \\
Q_{n+1}(x, t) & =E_{n}^{-1}\left\{\beta^{2} E_{2}\left[\frac{1}{x} \frac{\partial}{\partial x}\left(x \frac{\partial Q_{n}}{\partial x}\right)+P_{n} \frac{\partial Q_{n}}{\partial x}-2 x t Q_{n}-4 e^{t}\right]\right\}, \\
Q_{0}(x, t) & =x^{2} e^{t} .
\end{aligned}
$$


Then from as before we can find the first few components in the form

$$
\begin{aligned}
& \mathrm{P}_{0}(x, t)=x^{2} \mathrm{t}, \\
& \mathrm{P}_{1}(x, t)=E^{-1}\left\{\beta^{2} \mathrm{E}_{2}[0]\right\}=0, \\
& \mathrm{Q}_{0}(x, \mathrm{t})=x^{2} e^{\mathrm{t}}, \\
& \mathrm{Q}_{1}(x, \mathrm{t})=\mathrm{E}^{-1}\left\{\beta^{2} \mathrm{E}_{2}[0]\right\}=0,
\end{aligned}
$$

Then the exact solutions of a system (5.7) are

$$
\mathrm{P}(x, \mathrm{t})=\sum_{n=0}^{\infty} \mathrm{P}_{\mathrm{n}}(x, \mathrm{t})=x^{2} \mathrm{t}, \quad \mathrm{Q}(x, \mathrm{t})=\sum_{n=0}^{\infty} \mathrm{Q}_{n}(x, \mathrm{t})=x^{2} e^{\mathrm{t}}
$$

\section{Conclusion}

This paper examines the convergence of the new double transform, and explain the effectiveness and ease of the method used to solve the singular system of linear and nonlinear hyperbolic equations, as we obtained the exact solutions using only one step. Comparing this method with other methods, such as the Adomian method and the homotopy method, we find that this method is faster and easier for them to reach for exact solutions.

\section{Acknowledgment}

The authors extend their appreciation to the Deanship of Scientific Research at King Khalid University for funding this work though General Project under grant number (G.R.P-77-39).

\section{References}

[1] J. Biazar, H. Ghazvini, Hes variational iteration method for solving linear and non-linear systems of ordinary differential equations, Appl. Math. Comput., 191 (2007), 287-297. 1

[2] R. R. Dhunde, G. L. Waghmare, On Some Convergence Theorems of Double Laplace Transform, J. Inform. Math. Sci., 6 (2014), 45-54. 1

[3] T. M. Elzaki, Application of Projected Differential Transform Method on Nonlinear Partial Differential Equations with Proportional Delay in One Variable, World Appl. Sci. J., 30 (2014), 345-349. 1

[4] T. M. Elzaki, Double Laplace Variational Iteration Method for Solution of Nonlinear Convolution Partial Differential Equations, Arch. Sci., 65 (2012), 588-593. 1

[5] T. M. Elzaki, J. Biazar, Homotopy Perturbation Method and Elzaki Transform for Solving System of Nonlinear Partial Differential Equations, World Appl. Sci. J., 24 (2013), 944-948.

[6] T. M. Elzaki, S. M. Elzaki, E. A. Elnour, On the New Integral Transform Elzaki Transform Fundamental Properties Investigations and Applications, Global J. Math. Sci., 4 (2012), 1-13. 1

[7] T. M. Elzaki, E. M. A. Hilal, Solution of Telegraph Equation by Modified of Double Sumudu Transform Elzaki Transform, Math. Theory Model., 2 (2012), 95-103. 1

[8] J.-H. He, Variational iteration method for delay differential equations, Commun. Nonlinear Sci. Numer. Simul., 2 (1997), 235-236. 1

[9] J.-H. He, Variational iteration method a kind of non-linear analytical technique: some examples, Int. J. Non-linear Mech., 34 (1999), 699-708.

[10] J.-H. He, X.-H. Wu, Variational iteration method: new development and applications, Comput. Math. Appl., 54 (2007), 881-894. 1

[11] A. A. Hemeda, New iterative method: an application for solving fractional physical differential equations, Abstr. Appl. Anal., 2013 (2013), 9 pages. 1

[12] E. Hesameddini, H. Latifizadeh, Reconstruction of variational iteration algorithms using the Laplace transform, Int. J. Nonlinear Sci. Num. Simul., 10 (2009), 1377-1382. 1

[13] S. A. Khuri, A. Sayfy, A Laplace variational iteration strategy for the solution of differential equations, Appl. Math. Lett., 25 (2012), 2298-2305. 1 
[14] K. M. Saad, Comparing the Caputo, Caputo-Fabrizio and Atangana-Baleanu derivative with fractional order: Fractional cubic isothermal auto-catalytic chemical system, Eur. Phys. J. Plus, 133 (2018), 94 pages. 1

[15] K. M. Saad, E. H. Al-Shareef, M. S. Mohamed, X. J. Yang, Optimal q-homotopy analysis method for time-space fractional gas dynamics equation, Eur. Phys. J. Plus, 132 (2017), 23 pages.

[16] K. M. Saad, E. H. F. Al-Sharif, Analytical study for time and time-space fractional Burgers' equation, Adv. Differ. Equ., 2017 (2017), 300 pages.

[17] K. M. Saad, A. A. AL-Shomrani, An application of homotopy analysis transform method for Riccati differential equation of fractional order, J. Fract. Calc. Appl., 7 (2016), 61-72.

[18] K. M. Saad, D. Baleanu, A. Atangana, New fractional derivatives applied to the Kortewegde Vries and Kortewegde VriesBurgers equations, Comp. Appl. Math., 2018 (2018), 14 pages. 1

[19] G.-C. Wu, Challenge in the variational iteration method-a new approach to identification of the Lagrange mutipliers, J. King Saud Univ. Sci., 25 (2013), 175-178. 1

[20] G.-C. Wu, Laplace transform Overcoming Principle Drawbacks in Application of the Variational Iteration Method to Fractional Heat Equations, Therm. Sci., 16 (2012), 1257-1261.

[21] G.-C. Wu, Variational iteration method for solving the time-fractional diffusion equations in porous medium, Chin. Phys. B, 21 (2012), 120504. 1

[22] G.-C. Wu, D. Baleanu, Variational iteration method for fractional calculus-a universal approach by Laplace transform, Adv. Differ. Equ., 2013 (2013), 18-27. 1

[23] G.-C. Wu, D. Baleanu, Variational iteration method for the Burgers' flow with fractional derivatives-New Lagrange multipliers, Appl. Math. Model., 37 (2012), 6183-6190. 1 